\title{
Activity-dependent gating of lateral inhibition by correlated mitral cell activity in the mouse main olfactory bulb Armen C Arevian ${ }^{1,3}$ and Nathaniel N Urban*1,2,3
}

\author{
Address: ${ }^{1}$ Center for Neuroscience, University of Pittsburgh, Pittsburgh, PA, USA, ${ }^{2}$ Dept. of Biology, Carnegie Mellon University, Pittsburgh, PA, \\ USA and ${ }^{3}$ Center for the Neural Basis of Cognition, Pittsburgh, PA, USA \\ Email: Nathaniel N Urban* - nurban@cmu.edu \\ * Corresponding author
}

from Sixteenth Annual Computational Neuroscience Meeting: CNS*2007

Toronto, Canada. 7-12 July 2007

Published: 6 July 2007

BMC Neuroscience 2007, 8(Suppl 2):PI26 doi:I0.1 I86/I47|-2202-8-S2-PI26

(c) 2007 Arevian and Urban; licensee BioMed Central Ltd.

Excitatory mitral and tufted cells $(\mathrm{M} / \mathrm{Ts})$ provide the primary output of the mouse main olfactory bulb (MOB). M/ Ts provide excitatory input to and receive inhibitory input from GCs via the dendrodendritic synaptic connections. These circuits provide both recurrent and lateral inhibition among $\mathrm{M} / \mathrm{Ts}$. However, given the large area spanned by $\mathrm{M} / \mathrm{T}$ secondary dendrites as well as the lack of evidence for a clear correlation between the proximity of $\mathrm{M} / \mathrm{Ts}$ and their odor response profiles, we asked what mechanism could provide for specific and useful lateral inhibitory connectivity? To address this question we conducted whole-cell patch clamp recordings of pairs of $\mathrm{M} / \mathrm{Ts}$ in the MOB. Current steps (400 ms, 0-1200 pA) were injected into one of the paired cells (Cell A). We then compared the firing rate of Cell A when it was stimulated alone vs. when it was stimulated during simultaneous activation of a second $\mathrm{M} / \mathrm{T}$ (Cell B) at approximately $80 \mathrm{~Hz}$. We found that activity of Cell $\mathrm{B}$ significantly reduced the firing rate of Cell A only when Cell A was firing at frequencies between 35 and $110 \mathrm{~Hz}(19 \% / 17 \mathrm{~Hz}$ peak reduction, $\mathrm{n}=$ 16 pairs, $\mathrm{p}<0.05)$. This effect, which we call activitydependent lateral inhibition, is presumably due to activation of GCs correlated M/T cell activity and subsequent saturation of GC output. Furthermore, activation of larger populations of presynaptic $\mathrm{M} / \mathrm{Ts}$ via extracellular stimulation in the glomerular layer produced similar activitydependent lateral inhibition but of higher magnitude and occurring at lower frequencies $(25 \%$ peak reduction between postsynaptic firing rates between 25 and $65 \mathrm{~Hz}$, $\mathrm{n}=8, \mathrm{p}<0.05)$. We then implemented this physiologi- cally characterized mechanism in a network model with all-to-all connectivity. Results show that initially correlated patterns of activity are decorrelated in a spatially independent manner using this activity-dependent mechanism. These results suggest that the magnitude of inhibition received by $\mathrm{M} / \mathrm{Ts}$ is dynamically determined based on the pattern of activity within the bulb and can be used to decorrelate similar input patterns, enhancing odor discrimination. Supported by R01 - DC005798. 